\title{
DIAGNÓSTICOS DE ENFERMAGEM EM GESTANTES DE ALTO RISCO: AS NECESSIDADES PSICOSSOCIAIS EM FOCO
}

Celma Aparecida Barbosa dos Santos ${ }^{1}$ Janaina Cristina Pasquini de Almeida ${ }^{1}$ Letícia Yamawaka de Almeida ${ }^{1}$ Jaqueline Lemos de Oliveira ${ }^{1}$ Vanessa Pellegrino Toledo ${ }^{1}$

Jacqueline de Souza

\begin{abstract}
https://orcid.org/0000-0002-5112-1385 https://orcid.org/0000-0003-0331-0365 https://orcid.org/0000-0002-5192-6052 https://orcid.org/0000-0003-3699-0280 https://orcid.org/0000-0003-4009-1042 https://orcid.org/0000-0002-6094-6012
\end{abstract}

Objetivo: Identificar as necessidades psicossociais referidas nos estudos sobre gestação de alto risco buscando os diagnósticos correspondentes na Classificação Internacional das Práticas de Enfermagem em Saúde Coletiva (CIPESC®). Método: Estudo qualitativo de revisão narrativa. Inicialmente. realizou-se um levantamento bibliográfico na base de dados Literatura Latino-Americana e do Caribe em Ciências da Saúde (LILACS). Após leitura e organização dos dados, os aspectos psicossociais abordados nos estudos foram listados e relacionados com os diagnósticos da CIPESC@ correspondentes. Resultados: Fatores como relação profissional-paciente, reações emocionais negativas, redes de apoio, insegurança, aceitação e conflitos apresentavam correspondentes especificos na CIPESC®. Em contrapartida, circunstâncias que se caracterizavam como riscos psicossociais a estas gestantes não tinham diagnósticos correspondentes, sendo abordadas de forma abrangente nesta classificação. Conclusão: O presente estudo reforça a complexidade do uso de classificações no âmbito psicossocial e convida os profissionais e pesquisadores a repensarem seus planos de cuidado e práticas assistenciais às gestantes de alto risco

Descritores: Gravidez de alto risco; Diagnóstico de enfermagem; Saúde Mental.

\section{NURSING DIAGNOSES IN HIGH-RISK PREGNANT WOMEN: THE PSYCHOSOCIAL NEEDS IN FOCUS.}

Objective: To identify the psychosocial needs mentioned in studies on high-risk pregnancies seeking the corresponding diagnoses in the International Classification of Nursing Practices in Public Health (CIPESC $®$ ). Method: Qualitative study of narrative review. Initially, a bibliographic survey was carried out in the database "Latin American and Caribbean Literature in Health Sciences (LILACS)". After reading and organizing the data, the psychosocial aspects covered in the studies were listed and related to the corresponding CIPESC $®$ diagnoses. Results: Factors such as professional-patient relationship, negative emotional reactions, support networks, insecurity, acceptance and conflicts had specific correspondents CIPESC®. On the other hand, circumstances that were characterized as psychosocial risks to these pregnant women did not have corresponding diagnoses, being addressed comprehensively in this classification. Conclusion: Present study reinforces the complexity of the use of classifications in the psychosocial context and invites professionals and researchers to rethink their care plans and care practices for high-risk pregnant women

Descriptors: Pregnancy High-Risk; Nursing Diagnosis; Mental Health.

\section{DIAGNÓSTICOS DE ENFERMERÍA EN MUJERES EMBARAZADAS DE ALTO RIESGO: LAS NECESIDADES PSICOSOCIALES EN FOCO.}

Objetivo: Identificar las necesidades psicosociales a las que se refieren los estudios sobre embarazos de alto riesgo que buscan los diagnósticos correspondientes en la Clasificación Internacional de Prácticas de Enfermería en Salud Pública (CIPESC®). Método: Estudio cualitativo de la revisión narrativa. Inicialmente, se realizó una encuesta bibliográfica en la base de datos "Literatura latinoamericana y caribeña en ciencias de la salud (LILACS)". Después de leer y organizar los datos, se enumeraron los aspectos psicosociales abordados en los estudios y se relacionaron con los diagnósticos CIPESC@ correspondientes. Resultados: Factores como la relación profesional-paciente, reacciones emocionales negativas, redes de apoyo, inseguridad, aceptación y conflictos tuvieron corresponsales especificos en CIPESC®. Por otro lado, las circunstancias que se caracterizaron como riesgos psicosociales para estas mujeres embarazadas no tuvieron los diagnósticos correspondientes, y se abordaron de manera integral en esta clasificación. Conclusión: El presente estudio refuerza la complejidad del uso de clasificaciones en el contexto psicosocial e invita a profesionales e investigadores a repensar sus planes de atención y prácticas de atención para mujeres embarazadas de alto riesgo.

Descriptores: Embarazo de Alto Riesgo; Diagnóstico de Enfermería; Salud Mental.

${ }^{1}$ Escola de Enfermagem de Ribeirão Preto da Universidade de São Paulo, SP, Brasil. Autor correspondente: Jaqueline Lemos de Oliveira - Email: jaquelemos@usp.br Recebido: 25/02/2020 - Aceito: 11/06/2020 


\section{INTRODUÇÃO}

A gestação de alto risco consiste no processo de gravidez cujas mulheres apresentam maiores chances de evolução desfavorável para si e/ou para o feto em decorrência de alguma doença, agravo ou outros problemas prévios ou em curso no período do resultado positivo do exame de gravidez

A importância das necessidades psicossociais específicas do ciclo gravídico puerperal tem sido apontada por vários estudiosos da área que destacam a atenção redobrada que os profissionais devem ter em relação às gestantes de alto risco ${ }^{2-3}$

No que se refere à atenção básica e a saúde da mulher no ciclo gravídico de alto risco, a abordagem de Diagnósticos de Enfermagem relacionados às questões emocionais e psíquicas é extremamente necessária, haja vista a importância deste setting de cuidado tanto para o encaminhamento aos serviços especializados quanto para a garantia do acompanhamento de saúde de ambos, gestante e feto.

Neste sentido, as Classificações de Enfermagem são essenciais para que o enfermeiro oriente sua assistência e defina a melhor conduta a ser tomada, pois ao estabelecer o diagnóstico utilizando-se de alguma classificação oficial várias intervenções associadas ao mesmo são disponibilizadas propiciando ao profissional a instrumentalização de sua prática de acordo com as necessidades levantadas em sua avaliação ${ }^{4}$.

A Resolução № 358/2009 do Conselho Federal de Enfermagem (Cofen) dispõe sobre a Sistematização da Assistência de Enfermagem (SAE) e a implementação do Processo de Enfermagem nos diferentes locais de cuidado/assistência à saúde ${ }^{5}$, incluindo uma sequência de atividades, a saber coleta de dados e informações sobre o individuo assistido, avaliação das informações com a utilização de Diagnósticos de Enfermagem, elaboração de planejamentos das atividades assistenciais, implementação dos cuidados e avaliação dos resultados obtidos por meio das ações realizadas ${ }^{5}$.

É importante salientar que o modelo biológico e a prática baseada em evidências, influenciam esse sistema de classificação, e o resultado das Intervenções de Enfermagem é dirigido pelo diagnóstico, que em sua essência descreve formatos para estabelecer correlações estatísticas e padronizadas, bem como para implementar o desenvolvimento do pensamento crítico e do raciocínio clínico no processo de cuidar da gestante de alto risco ${ }^{6-7}$.

Diante da necessidade de uniformizar as ações da profissão e tornar mais clara a linguagem de trabalho nas várias instâncias de ação do enfermeiro surgem as clas- sificações de enfermagem. Embora a criação de algumas classificações não seja recente o uso das mesmas ainda se constitui um desafio para os enfermeiros, sobretudo na saúde coletiva ${ }^{8}$.

A North American Nursing Diagnosis Association (NANDA) é um sistema de classificação desenvolvida na década de 1970 para dar suporte ao julgamento clínico dos problemas potenciais ou reais de saúde e das condições dos indivíduos, famílias ou comunidades por meio de diagnósticos específicos?. Nesse sistema de classificação alguns diagnósticos aludem a aspectos psicossociais como auto percepção, enfrentamento/tolerância ao estresse e papéis e relacionamentos. No entanto, há uma ênfase nas condições biológicas/fisiológicas, sobretudo do contexto hospitalar, e no tocante à saúde mental, nos aspectos psiquiátricos em detrimento dos psicossociais.

No âmbito da Saúde Coletiva, a Associação Brasileira de Enfermagem (ABEn) em 1996 deu início a um projeto para a Classificação Internacional das Práticas de Enfermagem em Saúde Coletiva (CIPESC $\left.{ }^{8}\right)^{8}$. Neste sistema de classificação a disposição dos diagnósticos está dividida em dois blocos, as necessidades psicobiológicas e as psicossociais. As necessidades psicossociais são divididas em oito, a saber: segurança, liberdade, aprendizagem, gregária, recreação, autoestima, participação e autoimagem ${ }^{10}$.

Vale ressaltar que ambos os sistemas de classificação contêm diagnósticos relacionados à saúde mental, no entanto entende-se que os diagnósticos na NANDA estão pautados mais em aspectos psiquiátricos e na CIPESC $₫$ estes partem de uma noção de caráter mais psicossocial.

Ademais, é importante salientar que a CIPESC $\circledast$ é o único sistema desenvolvido até o momento com o tema exclusivo das necessidades psicossociais e com ações direcionadas especificamente para a população que utiliza a rede de serviços com uma abordagem voltada para a Saúde Coletiva no contexto de saúde brasileiro.

Os estudos relacionados à gestação de alto risco têm se baseado majoritariamente no cuidado biológico do ser humano ${ }^{11-24}$. Assim, considerando que as gestantes de alto risco apresentam maior vulnerabilidade e que, apesar de pouco explorado, os aspectos psicossociais permeiam todo o ciclo gestacional e devem ser abordados durante a Consulta de Enfermagem, a presente pesquisa teve como objetivo identificar as necessidades psicossociais referidas nos estudos sobre gestação de alto risco buscando os diagnósticos correspondentes na CIPESC ${ }^{\circledR}$.

Espera-se com este estudo discutir a pertinência dos diagnósticos deste sistema de classificação bem como o peso que os estudos da área têm dado para os aspectos 
psicossociais das mulheres na condição de gestante de alto risco.

\section{MÉTODO}

\section{Tipo de Estudo}

Trata-se de um estudo qualitativo de revisão narrativa, que tem por objetivo revisitar as produções bibliográficas de um determinado assunto, sob o ponto de vista teórico ou conceitual. Esta categoria de pesquisa, apesar de não possuir metodologia que permita a reprodução dos dados e/ou respostas quantitativas, é fundamental para a aquisição e atualização do conhecimento sobre uma determinada temática em um curto espaço de tempo ${ }^{25}$

Este trabalho constituiu a primeira fase de um projeto para o desenvolvimento de uma tecnologia de suporte para a SAE das gestantes de alto risco e parte de uma proposta do Cofen e da Coordenação de Aperfeiçoamento de Pessoal de Ensino Superior (CAPES) para o desenvolvimento de pesquisas científicas e tecnológicas com foco na SAE (Edital № 27/2016).

\section{Local do Estudo}

O processo de levantamento bibliográfico foi realizado de forma não sistemática e foram recuperados artigos indexados na base de dados Literatura Latino-Americana e do Caribe em Ciências da Saúde (LILACS), uma vez que, esta base abrange documentos técnicos científicos das áreas da saúde latino-americanos, o que possibilita uma análise da realidade do continente no qual a população em estudo está inserida, além de possuir acesso livre para possiveis leitores. Além disso, considerando que a presente revisão teve como foco a utilização da CIPESC® priorizou-se esta base entendendo que por meio dela seria possivel obter um panorama geral dos estudos sobre a temática no contexto brasileiro.

\section{Participantes do Estudo}

Para a busca considerou-se os seguintes descritores: "Gravidez de Alto Risco"; "Gestação de Alto Risco", "Gestante de Alto Risco". Destaca-se que os descritores supracitados foram utilizados em português e a combinação realizada com operadores booleanos "AND" e "OR". Foram incluídos os artigos na íntegra sobre a temática da gestação de alto risco, publicados em português, inglês ou espanhol, no período de 2013 a 2017.

\section{Coleta de Dados}

Para a elaboração desse estudo, a busca na literatura foi realizada entre o período de março a maio de 2018. Para organização dos dados utilizou-se um roteiro previamente elaborado contendo os seguintes itens: título e local do estudo, objetivos, ano, população, método, principais resultados, se o estudo fazia menção a algum aspecto psicossocial das gestantes e quais eram esses aspectos.

\section{Procedimentos de análise de dados}

Após as leituras realizadas para a extração dos dados dos artigos e o preenchimento do roteiro de coleta de dados, os achados foram alocados em planilhas no Excel ${ }^{\circledR}$. Primeiramente foi realizada uma classificação geral destacando os contextos nos quais os estudos foram realizados (hospital, atenção básica, especialidades etc.), o tipo dos estudos (qualitativo, quantitativo) e quantos deles faziam menção aos aspectos psicossociais.

Em seguida foram agrupados todos os artigos que mencionavam tais aspectos especificando também os contextos e abordagem metodológica. A fase seguinte da análise implicou na listagem de todos os aspectos psicossociais referidos em tais artigos, sendo identificados 94 aspectos que foram organizados em 17 categorias baseadas em critérios de similaridade.

A partir de então os dois pesquisadores envolvidos na análise dos dados examinaram cada aspecto psicossocial buscando identificar seus correspondentes no sistema de classificação adotado. Em seguida as implicações dos resultados obtidos foram discutidas com base na literatura científica.

\section{Procedimentos éticos}

O projeto foi submetido ao Comitê de Ética em Pesquisa da Escola de Enfermagem de Ribeirão Preto da Universidade de São Paulo e aprovado sob Parecer № 253/2018.

\section{RESULTADOS}

O levantamento resultou em $23^{11-24,26-34}$ artigos dos quais 1112-16,18,20-24 utilizaram método quantitativo, 10 11,26-34 qualitativo e dois ${ }^{17,19}$ relatos de caso. Dos 23 artigos 18 11-15,17-19,21,2324.26-31,33 foram realizados em contexto hospitalar os demais eram em ambulatórios especializados ${ }^{16,22,32,34}$ e Unidade Básica de Saúde ${ }^{20}$. Em relação aos aspectos abordados nos estudos, observou-se que a maioria deles $(n=14)^{11-24}$ esteve pautada em aspectos fisiopatológicos, como características clínico-obstétricas ${ }^{11,15-16,19-20}$, diagnósticos e patologias específicas ${ }^{12,14,17-18,23}$, padrão do consumo de medicamentos $^{13}$ e perfil epidemiológico $20-22,24$.

Dos nove estudos ${ }^{26-34}$ que referiram aspectos psicossociais sete foram desenvolvidos em contexto hospita$\operatorname{lar}^{26-31,33}$, os demais em ambulatórios especializados ${ }^{32,34}$ e 
todos utilizaram abordagens qualitativas. Tais aspectos bem como os diagnósticos correspondentes da CIPESC®, quando existentes, são apresentados no Quadro 1.

Conforme pode ser observado, alguns aspectos relacionados à relação profissional-paciente, às reações emocionais negativas, às redes de apoio, ao sentimento de insegurança, aceitação e conflitos apresentavam correspondentes específicos na CIPESC $\circledast$. Apesar disso, várias circunstâncias que se caracterizam como riscos psicossociais à estas gestantes não tinham diagnósticos correspondentes neste sistema de classificação.

Quadro 1 Aspectos psicossociais abordados nos estudos e os diagnósticos correspondentes na CIPESC®.

Relação profissional-paciente
Falha na comunicação com
profissionais, falta interação e
diálogo médico-paciente Tomada
de decisão unilateral
Dificuldade de adesão, falha para
atender a sequência correta do
pré-natal
Não acompanhamento adequado
do pré-natal, Falta de autonomia
da usuária
Diagnóstico Bl
Histórico de gestações

Parto traumático

História de aborto em gestação anterior, processo de luto

Transtornos mentais

Transtornos de ansiedade, bipolar ou psicóticos

Baby-Blues, depressão pós-parto transtorno depressivo

\section{Sintomas psiquicos}

Ideação paranoide, agitação psicomotora

Reações emocionais negativas

Ansiedade, angústia, apreensão, inquietações, tristeza, estresse, sofrimento

Diagnósticos A4 e A6

Medo

Temor, medo, medo da morte, medo relativo à via do parto, Medo de sofrer parada cardiaca, medo de algo acontecer com os filhos, pânico

\section{Aceitação}

Rejeição, revolta, negação, culpa, frustração, surpresa Descontentamento. desapontamento, não aceitação da interrupção da gravidez Dificuldade na aceitação do diagnóstico de alto risco Gravidez não planejada, gravidez não desejada

Diagnósticos G2, A2, 16.2

Dificuldade em conciliar o cuidado da casa, da família e de si

Tomada de decisão limitada, Autocuidado

\section{Aspectos sociais}

Trabalho estressante, desemprego, situação socioeconômica desfavorável Distanciamento do sistema de saúde, prejuizos no lazer Condições culturais e socioeconômicas deficitárias

Riscos psíquicos

Histórico de depressão. vulnerabilidade aos transtornos mentais

\section{Conflitos}

Situação conjugal instável, relacionamento conjugal insatisfatório

Conflitos, conflitos familiares Diagnósticos D2 ou D4

Preocupação

Tensão, preocupação, preocupação com o bebê, incerteza quanto ao porvir Preocupação da prematuridade do bebê, preocupação com os outros filhos
Déficit de autocuidado
Redes de apoio

Isolamento social, solidão, falta de apoio, falta de apoio do pai do bebê

Rede de apoio empobrecida

não ter espaço para conversar e

expressar-se

Religiosidade

Diagnósticos Al e Dl

Insegurança

Falta de confiança em si sentimento de incapacidade, impotência, insegurança Fragilidade emocional, baixa autoestima, não se ver em condições de ter o filho

Diagnósticos F1 ou F2

Informações

Dúvidas, déficit de informações,

desconhecimento dos riscos

Diagnósticos: $\mathrm{Bl}=$ Tomada de decisão comprometida; A4=Ansiedade decorrente do estado de saúde atual;

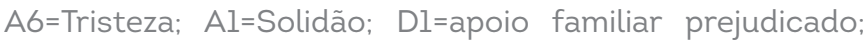
G2=Adaptação/enfrentamento inadequado; $A 2=A n s i e d a d e$ frente a coleta e resultado de exames; 16.2=Gravidez indesejada; D2=Relacionamento familiar conflituoso; D4=Relacionamento interpessoal comprometido; $\mathrm{Fl}=$ Autoestima prejudicada; F2=Autoestima baixa.

\section{DISCUSSÃO}

Identificaram-se que poucas pesquisas sobre a temática estudada foram desenvolvidas em contextos extra-hospitalares, apesar disso os estudos abordavam de modo abrangente as necessidades psicossociais das gestantes de alto risco corroborando para a melhoria da prática nos diferentes settings assistenciais.

As questões mais fortemente relacionadas aos sintomas e riscos de adoecimento psíquico como traumas de gestações prévias, sentimentos de medo, oscilações do humor, preocupações específicas com a condição da gestação e seus riscos, bem como o próprio transtorno mental concomitante à gestação não foram contempladas pela CIPESC®. Dado que tal sistema de classificação foi idealizado para o uso no contexto extra-hospitalar ${ }^{35}$ e que o acolhimento das demandas de saúde mental no âmbito comunitário seja uma preconização recente ${ }^{36-37}$, entende-se que estes fatos podem justificar a ausência de determinadas necessidades e diagnósticos no referido sistema. Além disso, o fato do termo "psicossocial" ter conotações cada vez mais abrangentes e uma conceituação não uniforme entre as diferentes especialidades ${ }^{38}$ pode também contribuir para os resultados identificados neste estudo. 
Por outro lado, problemas da relação profissional-paciente, a manifestação de emoções negativas, dificuldade de aceitação de sua condição, conflitos e as redes de apoio foram aspectos contemplados pela CIPESC®, com proposições importantes para a efetivação da assistência de enfermagem, resultado que corrobora a pertinência desse instrumento para a sistematização da assistência em diferentes contextos ${ }^{4,39}$

Aspectos relacionados ao autocuidado, alterações nos projetos de vida, impacto social e demandas por informações por parte das gestantes também foram reportados nos estudos, mas não tinham correspondentes na CIPESC®. Longe de constituir-se como uma crítica a este sistema de classificação, o presente estudo tanto reforça a complexidade do uso de classificações no âmbito da saúde mental, conforme já apontado em estudo prévio ${ }^{40}$, dada a dinâmica e subjetividade das pessoas frente às diferentes circunstâncias da vida, quanto convida os profissionais e pesquisadores a repensarem as necessidades que costumam abranger nos seus planos de cuidado a fim de ampliar suas perspectivas assistenciais no que diz respeito, sobretudo, às questões psicossociais de grupos específicos.

Por meio de um estudo que objetivou identificar evidências referentes à aplicação do processo de enfermagem no cuidado desenvolvido pelo enfermeiro no que tange às questões de saúde mental, é possivel conceber que há a necessidade de reorganização do processo de trabalho dos profissionais envolvidos na atenção oferecida à pacientes com questões psíquicas. Sendo assim, o Processo de Enfermagem pode possibilitar uma amplitude maior na avaliação do estado de saúde do paciente, pois é importante que o foco do cuidado se dirija ao reconhecimento do significado individual da experiência psíquica no seu contexto social, político e cultural, e não se restrinja apenas a sintomatologia física ${ }^{6,41}$

Outro ponto para considerar além da necessidade do enfermeiro em dispensar técnicas humanizadas durante a atenção a gestante de alto risco, é promover intervenções de enfermagem baseadas na singularidade, enfatizando seus desejos, opiniões, sentimentos, bem como, incluindo sua família. Neste sentido os Diagnósticos de Enfermagem podem favorecer uma assistência individualizada às mulheres, baseada no conhecimento científico e na obtenção de resultados com indicadores de qualidade, usando os sistemas de classificação, o que também facilita a comunicação entre a equipe de atenção $0^{7.42}$

Acrescenta-se que, em termos metodológicos, os estudos que utilizaram abordagens qualitativas parecem estar mais sensiveis a tais necessidades e podem, portanto, re- forçar necessidades e respectivas intervenções no âmbito acadêmico, contribuindo para futura ampliação do arcabouço de diagnósticos e intervenções dos instrumentos de classificação atuais.

\section{Limitações do estudo}

Em relação às limitações do presente estudo destacam-se dois aspectos: a busca realizada em apenas uma base de dados e a não utilização dos descritores em outras línguas. Entende-se que dado o uso da base LILACS, ao menos os correspondentes em espanhol já ampliariam o volume de estudos a serem revisados bem como proporcionariam um panorama mais amplo sobre as produções nessa temática.

\section{Contribuições para a prática}

Tendo em vista o caráter processual que a referida pesquisa assumiu e questões de exequibilidade, vale destacar que, apesar disso o presente estudo propiciou a elaboração de um panorama documental/técnico sólido para as determinadas etapas da pesquisa e para ampliar as discussões sobre a SAE sob a ótica psicossocial.

Nesse sentido, revela-se a necessidade de aprimoramento da organização do processo de trabalho dos profissionais, tendo em vista o enfoque aos aspectos psicossociais dos indivíduos, a fim de proporcionar um cuidado integral.

\section{CONCLUSÃO}

Destaca-se que a Assistência de Enfermagem deve ter seu foco na prestação de cuidados voltados não apenas às questões fisiológicas das gestantes de alto risco, mas também deve atentar-se às alterações presentes nas esferas emocional, social e espiritual, as quais precisam ser sentidas e avaliadas pelo enfermeiro na busca de um cuidado integral. Ressalta-se que a observação de fatores psicossociais da gestante de alto risco no decorrer das atividades de assistência ou durante uma consulta de enfermagem possibilita uma avaliação mais profunda das necessidades da usuária, bem como norteia a elaboração de um planejamento de cuidado direcionado à finalidade de melhorar os fatores emocionais que possam estar afetando nesse periodo. Além disso, estudos sobre tais necessidades são cruciais para futura ampliação do arcabouço de diagnósticos e intervenções dos instrumentos de classificação atuais.

\section{Contribuições dos autores:}

Santos CAB e Souza J contribuíram na concepção e planejamento do projeto de pesquisa, obtenção, análise e 
interpretação dos dados, redação, revisão crítica e aprovação da versão final a ser publicada. Almeida JCP, Almeida LY, Oliveira JL e Toledo VP contribuíram com a obtenção, análise e interpretação dos dados, redação e aprovação da versão final a ser publicada.

FInanciamento: Projeto do Edital 27/2016 - proposta do Cofen e da Coordenação de Aperfeiçoamento de Pesso- al de Ensino Superior (CAPES) para o desenvolvimento de pesquisas científicas e tecnológicas com foco na SAE.

Agradecimentos: Ao Instituto Federal de Educação, Ciência e Tecnologia do Sul de Minas Gerais - IFSULDEMINAS/Campus Poços de Caldas, ao Conselho Federal de Enfermagem - Cofen e à Coordenação de Aperfeiçoamento de Pessoal de Ensino Superior - CAPES.

\section{REFERÊNCIAS}

1. Ministério da Saúde (BR). Secretaria de Atenção à Saúde. Departamento de Ações Programáticas Estratégicas. Gestação de alto risco: manual técnico. Ministério da Saúde, Secretaria de Atenção à Saúde, Departamento de Ações Programáticas Estratégicas. - 5. ed. - Brasilia: Editora do Ministério da Saúde; 2012. [Internet]. 2010 [cited 2019 May 02]. Available from: https://bvsms.saude.gov.br/bvs/publicacoes/manual_tecnico_gestacao_alto_risco.pdf

2. Amorim TV, Souza IEO, Moura MAV, Queiroz ABA, Salimena AMO. Perspectivas do cuidado de enfermagem na gestação de alto risco: revisão integrativa. Enferm glob [Internet]. 2017 [cited 2019 May 02]; 46:51529. Available from: http://scielo.isciii.es/pdf/eg/v16n46/pt_1695-6141eg-16-46-00500.pdf.

3. Lindqvist M, Lindkvist M, Eurenius E, Persson M, Mogren I. Change of lifestyle habits-motivation and ability reported by pregnant women in northern Sweden. Sex Reprod Healthc [Internet]. 2017 [cited 2019 May 02]; 13:83-90. Available from: DOI: 10.1016/j.srhc.2017.07.001.

4. Nichiata LYI, Padoveze MC, Ciosak SI, Gryschek ALFPL, Costa AA, Takahashi RF et al. Classificação Internacional das Práticas de Enfermagem em Saúde Coletiva - CIPESC®: instrumento pedagógico de investigação epidemiológica. Rev Esc Enferm USP [Internet]. 2012 [cited 2019 Apr 25]; 46(3):766-71. Available from: http://dx.doi.org/10.1590/S008062342012000300032

5. Conselho Federal de Enfermagem (Cofen). Resolução № 358/2009. Dispõe sobre a Sistematização da Assistência de Enfermagem e a implementação do Processo de Enfermagem em ambientes, públicos ou privados, em que ocorre o cuidado profissional de Enfermagem. Diário Oficial da União, Brasilia (2009 out 15). [Internet]. 2009 [cited 2019 May 02]. Available from: http://www.cofen.gov.br/resoluo-cofen-3582009_4384. html

6. Garcia APRF, Freitas MIP, Lamas JLT, Toledo VP. Nursing process in mental health: an integrative literature review. Rev Bras Enferm [Internet]. 2017 [cited 2019 Apr 02]; 70(1):209-18. Available from: DOI: http:// dx.doi.org/10.1590/0034-7167-2016-0031.

7. Vergara Escobar OJ, Pulido Acuña GP, Montenegro Ramírez JD, González Salazar CA, Ortiz Acosta YJ. El lenguaje estandarizado enfermero: conocimiento y perspectiva para el cuidado materno-perinatal. Investig Enferm Imagen Desarr [Internet]. 2019 [cited 2020 Jan 02]; 21(1):1-11. Available from: https://doi.org/10.11144/Javeriana.ie21-1.elee

8. Batista L. Elaboração de roteiro de sistematização da assistência de enfermagem na atenção à gestante: proposta de utilização do CIPESC@. [Dissertação] Ribeirão Preto: Escola de Enfermagem de Ribeirão Preto/ USP, 2017. [Internet]. 2017 [cited 2019 May 02]. Available from: https:// www.teses.usp.br/teses/disponiveis/22/22134/tde-28022018-201121/ publico/LUCIANABATISTA.pdf
9. Herdman TH, Kamitsuru S. Diagnósticos de enfermagem da NANDA-I: definições e classificação 2018-2020 [NANDA International]. Brasil VV. 11. ed. Porto Alegre: Artmed; 2018

10. Cavalcante MDMA, Larocca LM, Chaves MMN, Cubas MR, Piosiadlo LCM, Mazza VA. Nursing terminology as a work process instrument of nurses in collective health. Rev Esc Enferm USP [Internet]. 2016 [cited 2019 May 02]; 50(4):607-613. Available from: DOI: http://dx.doi. org/10.1590/S0080-623420160000500010.

11. Azevedo RO, Silvino ZR, Ferreira HC. Diretrizes de enfermagem para assistência à gestante de alto risco: estudo descritivo. Online Braz J Nurs [Internet]. 2013 [cited 2019 May 08]; 12(Sup):623-5. Available from: http:// www.objnursing.uff.br/index.php/nursing/article/view/4512/html_2.

12. Magalhães MC, Raymundo CE, Bustamante-Teixeira MT. Morbidade materna extremamente grave a partir dos registros de internação hospitalar do Sistema Único de Saúde: algoritmo para identificação dos casos. Rev Bras Saude Mater Infant. [Internet]. 2013 [cited 2020 May 31] 13(1):17-22. Available from: http://www.scielo.br/scielo.php?script=sci arttextEpid=S1519-38292013000100002\&lng=en

13. Araújo DD, Leal MM, Santos EJV, Leal LB. Consumption of medicines in high-risk pregnancy: evaluation of determinants related to the use of prescription drugs and self-medication. Braz J Pharm Sci [Internet]. 2013 [cited 2020 May 31]; 49(3):491-499. Available from: http://www.scielo.br/ scielo.php?script=sci_arttext\&pid=S1984-82502013000300010\&lng=en.

14. Xavier RB, Bonan C, Martisn AC, Silva KS. Riscos reprodutivos e cuidados integrais de gestantes com sindromes hipertensivas: estudo transversal. Online braz j nurs [Internet]. 2013 [cited 2019 May 31]; 12(4):82333. Available from: http://www.objnursing.uff.br/index.php/nursing/ article/view/4249/html_1l.

15. Viera Neta FA, Crisóstomo VL, Castro RCMB, Pessoa SMF, Aragão MMS, Calou CGP. Avaliação do perfil e dos cuidados no pré-natal de mulheres com diabetes mellitus gestacional. Rev Rene [Internet]. 2014 [cited 2019 May 31]; 15(5):823-31. Available from: DOI: https://doi. org/10.15253/2175-6783.2014000500012.

16. Evangelista DR, Moura ERF, Costa CBJS, Bezerra CG, Valente MMOP Sousa CSP. Conhecimento e prática anticoncepcional de mulheres portadoras de Diabetes Mellitus. Esc Anna Nery [Internet]. 2014 [cited 2020 May 31]; 18(3):441-447. Available from: http://www.scielo.br/scielo. php?script=sci_arttext\&pid=S1414-81452014000300441\&lng=en

17. Conti FB, Hibner S, Costa TH, Dezan MR, Aravechia MG, Pereira RAA et al. Sucesso no manejo da trombocitopenia aloimune neonatal na segunda gestação: relato de caso. Einstein (São Paulo) [Internet]. 2014 [cited 2020 May 31]; 12(1): 96-99. Available from: http://www.scielo.br/scielo. php?script=sci_arttext\&pid=S1679-45082014000100018\&lng=en.

18. Fernandes LB, Amaral WN. Clinical study of skin changes in low and 
high risk pregnant women. An Bras Dermatol [Internet]. 2015 [cited 2019 Apr 10]; 90(6):822-6. Available from: DOI: 10.1590/abd18064841.20153570.

19. Barbosa RR, Mario ADS, Calil OA, Jacques TM, Serpa RG, Barbosa LFM. Acompanhamento de Gestante Portadora de Sindrome de Marfan com Tubo Valvado Metálico Aórtico. Int j cardiovasc sci [Internet]; 2016 [cited 2019 Apr 10]; 29(4):f:329-l:332. Available from: https://pesquisa. bvsalud.org/portal/resource/\%20es/biblio-831875.

20. Melo WA, Alves JI, Ferreira AAS, Souza VS, Maran E. Gestação de alto risco: fatores associados em município do noroeste paranaense. Espaço para a Saúde - Revista de Saúde Pública do Paraná [Internet]. 2016 [cited 2019 Apr 25]; 17(1):83-92. Available from: DOI: http://dx.doi. org/10.22421/1517-7130.2016v17nlp83

21. Silva TC, Varela PLR, Oliveira RR, Mathias TAF. Morbidade materna grave identificada no Sistema de Informações Hospitalares do Sistema Único de Saúde, no estado do Paraná, 2010. Epidemiol Serv Saúde [Internet]. 2016 Sep [cited 2020 May 31]; 25(3):617-628. Available from: http://www.scielo.br/scielo.php?script=sci_arttext\&pid=S2237-96222016000300617\&lng=en.

22. Costa LD, Cura CC, Perondi AR, França VF, Bortoloti DS. Perfil epidemiológico de gestantes de alto risco. Cogitare Enferm [Internet]. 2016 [cited 2019 Apr 25]; 21(2):01-08. Available from: DOI: http://dx.doi. org/10.5380/ce.v2li2.44192.

23. Coli M, Zani AV. Validação de um plano de alta de enfermagem para gestantes e puérperas de alto risco. Rev Min Enferm [Internet]. 2016 [cited 2019 Apr 25]; 20:e934. Available from: DOI: 10.5935/14152762.20160004 .

24. Azevedo RO, Ferreira HC, Silvino ZR, Christovam BP. Perfil das gestantes de alto risco hospitalizadas em uma maternidade: estudo descritivo. Online braz j nurs [Internet]. 2017 [cited 2019 Apr 25]; 16(2):218-225. Available from: https://pesquisa.bvsalud.org/portal/resource/pt/biblio-877012.

25. Rother ET. Revisão sistemática X revisão narrativa. Acta paul Enferm. [Internet]. 2007 [cited 2019 Apr 28]; 20(2):v-vi. Available from: https:// doi.org/10.1590/s0103-21002007000200001.

26. Bomfim OL, Coser O, Moreira MEL. Unexpected diagnosis of fetal malformations: therapeutic itineraries. Physis [Internet]. 2014 [cited 2019 Apr 25]; 24(2):607-622. Available from: http://dx.doi.org/10.1590/ S0103-73312014000200015.

27. Amorim TV, Souza IEO, Salimena AMO, Carvalho ALO, Silva LF, Langendorf TF. Promoção da saúde materna a partir do vivido do parto de mulheres cardiopatas. Cogitare enferm [Internet]. 2017 [cited 2019 Apr 25]; 4(22):e51641. Available from: https://revistas.ufpr.br/cogitare/article/view/51641/pdf.

28.Arrais AR, Mourão MA, Fragalle B. O pré-natal psicológico como programa de prevenção à depressão pós-parto. Saúde Soc [Internet]. 2014 [cited 2019 Apr 28]; 23(1):251-264. Available from: http://dx.doi. org/10.1590/S0104-12902014000100020.

29. Roque ATF, Carraro TE. Narrativas sobre a experiência de ser puérpera de alto risco. Esc Anna Nery [Internet]. 2015 [cited 2019 Apr 28]; 19(2):272-278. Available from: DOI: 10.5935/1414-8145.20150036.

30. Oliveira DC, Mandú ENT. Mulheres com gravidez de maior risco: vivências e percepções de necessidades e cuidado. Esc. Anna Nery [Internet]. 2015 [cited 2019 Apr 28]; 19(1):93-101. Available from: DOI: $10.5935 / 1414-8145.20150013$
31. Schmalfuss JM, Bonilha ALL. Implicações das restrições alimentares na vida diária de mulheres com diabete melito gestacional. Rev enferm UERJ [Internet]. 2015 [cited 2019 Apr 28]; 23(1):39-44. Available from: DOI: https://doi.org/10.12957/reuerj.2015.5712.

32.Amorim TV, Souza IEO, Salimena AMO, Melo MCSC, Paiva ACPC Moura MAV. Cotidiano da gravidez de risco por cardiopatia: estudo fenomenológico das relações assistenciais. Esc Anna Nery [Internet]. 2016 [cited 2019 Apr 28]; 20(4):e20160091. Available from: DOI: 10.5935/14148145.20160091 .

33. Langaro F, Santos AH. Adesão ao Tratamento em Gestação de Alto Risco. Psicol ciênc prof. [Internet]. 2014 [cited 2019 Apr 28]; 34(3):625-642. Available from: https://dx.doi.org/10.1590/1982-3703000782013.

34.Costa LD, Perondi AR, Cavalheiri JC, Ferreira AS, Teixeira GT, Bortoloti DS. Adequação do pré-natal de alto risco em um hospital de referência. Rev Rene. [Internet]. 2016 [cited 2019 Apr 28]; 17(4):459-65. Available from: DOI: 10.1.15253/2175-6783.2016000400004.

35. Cubas MR, Egry EY. Classificação Internacional de Práticas de Enfermagem em Saúde Coletiva - CIPESC $®$. Rev Esc Enferm. USP. [Internet]. 2008 [cited 2019 Apr 28]; 42(1):181-186. Available from: http://dx.doi.org/10.1590/ S0080-62342008000100024.

36. Clementino FS, Miranda FAN, Pessoa Júnior JM, Marcolino EC, Silva Júnior JA, Brandão GCG. Atendimento integral e comunitário em saúde mental: avanços e desafios da reforma psiquiátrica. Trab educ saúde. [Internet]. 2019 [cited 2019 Apr 28]; 17(1):e0017713. Available from: https://dx. doi.org/10.1590/1981-7746-sol00177

37. Pinheiro CW, Araújo MAM, Rolim KMC, Oliveira CM, Alencar AB. Teoria das relações interpessoais: reflexões acerca da função terapêutica do enfermeiro em saúde mental. Enferm. Foco [Internet] 2019 [cited 2020 Jun 08]:10(3):64-69. Available from: http://revista.cofen.gov.br/index.php/enfermagem/article/view/2291/580

38

Felix TS. Concepções do psicossocial e a política de saúde mental brasileira. [Dissertação] Fortaleza: Universidade Federal do Ceará, 2017. [Internet]. 2017 [cited 2019 May 02]. Available from: http://www.repositorio. ufc.br/bitstream/riufc/33105/3/2017_dis_tsfelix.pdf

39. Alves KYA, Dantas CN, Salvador PTCO, Dantas RAN. Vivenciando a classificação internacional de práticas de enfermagem em saúde coletiva: relato de experiência. Esc Anna Nery [Internet]. 2013 [cited 2019 Apr 28]: 17(2):381-388. Available from: http://dx.doi.org/10.1590/S141481452013000200025

40. Tavares CM. Mesquita LM. Sistematização da assistência de enfermagem e clinica ampliada: desafios para o ensino de saúde mental. Enferm Foco [Internet]. 2019 [cited 2020 Jan 4]; 10(7):121-126. Available from: http://revista.cofen.gov.br/index.php/enfermagem/article/ view/2810/560

41. Tarouco VS, Piexak DR, Santana CS, Oliveira ACC, Pinho LA, Martins KP. Cuidados de enfermagem frente a dimensão espiritual no periodo gestacional. Enferm Foco [Internet]. 2019 [cited 2020 Jan 4]; 10(5):185-190. Available from: http://revista.cofen.gov.br/index.php/enfermagem/article/ view/2688/688

42.Linch GFC, Paz AA, Caregnato RCA, Abreu AM, Souza EN. Ações coordenadas para implantação e consolidação da sistematização da assistência de enfermagem em um complexo hospitalar. Enferm Foco [Internet]. 2019 [cited 2020 Jan 3]; 10(4):82-88. Available from: http://revista.cofen. gov.br/index.php/enfermagem/article/view/2342/556. 